\title{
Cloning and expression of porA gene as the first step of a vaccine candidate study against Neisseria meningitidis serogroup A infection
}

\author{
Afrough $\mathrm{P}^{1,2}$, Behrouzi $\mathrm{A}^{1,2}$, Davari $\mathrm{M}^{1}$, Malekan $\mathrm{Ma}^{2}$, Fateh $\mathrm{A}^{1,2}$, Vaziri $\mathrm{F}^{1,2}$, Siadat $\mathrm{SD}^{1,2^{*}}$ \\ ${ }^{1}$ Mycobacteriology and Pulmonary Research Department, Pasteur Institute of Iran. Tehran, Iran. \\ ${ }^{2}$ Microbiology Research Center (MRC), Pasteur Institute of Iran, Tehran, Iran.
}

\begin{abstract}
Introduction: Neisseria meningitidis is a major causative agent of bacterial septicemia and meningitis in human. PorA is a major component of the outer membrane of $N$. meningitidis and functions as a cationic Porin. This study aimed to clone and determine the expression of PorA as the first step for producing a proper antigen in a vaccine study against N. meningitidis. Methods: An approximately 1200-bp fragment of porA gene was amplified by PCR using $N$. meningitidis serogroup A genomic DNA and then cloned into prokaryotic expression vector pET-28a. The resulting construct (pET28a-porA plasmid) was transformed into competent E.coli BL21 cells for expression of recombinant protein. The proper overexpression of the recombinant protein was verified by SDS-PAGE and Western Blotting. Results: Cloning of porA was confirmed by colony-PCR and enzymatic digestion. The nucleotide sequence homology of the cloned porA gene was 97\% , compared to the reference gene (NCBI GenBank accession number AL157959.1). The prokaryotic expression system (pET28a-porA- BL21) could produce our 45-kDa target recombinant protein, efficiently. Conclusion: The prokaryotic expression system and conditions used in this study provides an applicable method for producing recombinant PorA and possibly many other bacterial outer membrane proteins for future vaccine studies.
\end{abstract}

KEYWORDS: Neisseria meningitidis, PorA, pET-28a.

\section{INTRODUCTION}

Meningitis and sepsis are two extremely serious and lifethreatening diseases that still cause a significant morbidity and mortality in both children and adults. Meningococcal diseases can develop rapidly which may cause death or serious and permanent problems in less than 24 hours, even in those undergoing antibiotic treatments [1]. Neisseria meningitidis is a major cause of these diseases that is divided into 13 different serogroups based on the differences in its capsule polysaccharide structure. Serogroups A, B, C, Y and W135 are primary pathogens in human with different geographical distributions in different regions. For instance, serogroup A has caused epidemics in Asia and Africa, serogroup C in Europe and serogroup Y and W135 in the United States. Interestingly, the serogroup B infections in New Zealand indicates the potential of this bacterium to cause epidemics [2]. A number of factors, including a sporadic nature, a sudden onset, an

*Corresponding Author: Seyed Davar Siadat, Department of Mycobactriology and Pulmonary Research, Pasteur Institute of Iran, Tehran, Iran; Microbiology Research Center (MRC), Pasteur Institute of Iran, Tehran, Iran.

Email: d.siadat@gmail.com

Tel/Fax: (+98) 2166968853 antibiotic resistance (especially to ciprofloxacin) [3] and a rapid and severe progression, makes the vaccination necessary for meningococcal diseases as a control measure against the pathogen. The proteins Opa, Rmp, LbpA, TbpA, NhhA, NspA and NadA and the porins PorA and PorB are major meningococcal outer membrane $(\mathrm{OM})$ proteins $[4,5]$ which have been used as vaccine candidates in many studies. Among these proteins, the highest bactericidal antibody response has been induced against PorA that explains the rationale for most outer membrane vesicle (OMV)-based vaccines against meningococcal to be based on PorA; hence, this antigen was selected for this study [6, 7]. PorA with Mw of 42-45 kDa is an intramembrane cationic protein that is expressed in all the strains as a major component of the meningococcal OM $[8,9]$. Amino acid sequences of this protein are relatively conserved among different strains and are composed of 8 hydrophilic extracellular loops $[9,10]$. Two of these loops possess highly immunogenic properties (loop1 and loop4) and stimulate immune responses to induce the production of antibodies. The present study was conducted to use an expression system for producing recombinant PorA protein (rPorA) in E. coli host as the first step of a vaccine study against $\mathrm{N}$. meningitidis. It is expected that the potential immunogenicity of this recombinant 
protein in the presence of adjuvant compounds such as OMV and industrial adjuvants such as alum will be evaluated using an animal model [11-13].

\section{MATERIALS and METHODS}

Preparation of N. meningitides strains and vectors The lyophilized N. meningitides strain (ATCC CSBPI G243) was obtained from the Microbiology Department of Pasteur Institute of Iran and was restored in chocolate agar and Mueller-Hinton agar. E. coli BL21 (Novagen, Wisconsin, USA) strain was used for cloning and expression. pET-28a (Novagen, Wisconsin, USA) was used as the expression vector.

\section{Genome purification and PCR}

The genomic DNA from $N$. meningitidis serogroup A was extracted using the Genomic DNA Purification Kit (DNA Technology, Russia) based on the manufacturer's instructions. The concentration and purity of the genomic DNA was assessed using Nano Drop spectrophotometer (JENWAY 6305, UK). To amplify the desired gene fragment from the purified genome of the intended bacterial strain, the forward and the reverse primers were first designed with two restriction sites (i.e. HindIII and NcoI, respectively), so that their restriction sites were also available on the vector. The sequences of the forward and the reverse primers are as follows:

$\begin{array}{llll}\text { porA } & \text { (Forward HindIII): } & 5 & \text { 'CCGAAGCTT } \\ \text { GACGGATCCATGCGAAAAAAACTTACC 3' } \\ \text { porA (Reverse NcoI): 5 ' }\end{array}$
ATACTCGAGTTAGAATTTGTGGCGCAAACC3'

PCR was performed in $50 \mu \mathrm{l}$ reactions using $10 \mathrm{pm}$ forward and reverse primers and 1 unit of DNA polymerase enzyme (Sinagene, Tehran, Iran). The reaction was performed in the thermo cycler (Eppendorf, Germany) by first denaturation at $94^{\circ} \mathrm{C}$ for $3 \mathrm{~min}$, followed by 30 cycles of $94^{\circ} \mathrm{C}$ for $45 \mathrm{~s}, 55^{\circ} \mathrm{C}$ for $30 \mathrm{~s}$ and $72^{\circ} \mathrm{C}$ for $90 \mathrm{~s}$. The final extension was $5 \mathrm{~min}$ at $72^{\circ} \mathrm{C}$. The PCR product was visualized on $1.5 \%$ agarose gel. The exact size of the amplicon was expected to be $1196 \mathrm{bp}$.

\section{Cloning of porA gene}

After amplification of the gene fragment, the PCR product and pET-28a expression vector were digested by HindIII and NcoI restriction enzymes. The digestion product was electrophoresed on $1.5 \%$ agarose gel and purified from the gel. The ligation reaction containing the double-digested insert and the expression vector, was performed in the presence of 1 unit of T4 DNA Ligase (Thermo Fisher Scientific, Boston, MA, USA) and the resulting mixture was transformed into competent E. coli BL21 (Novagen, Wisconsin, USA). The transformed bacteria were inoculated on LB (Merck, Darmstadt, Germany) agar plate containing kanamycin $(50 \mu \mathrm{g} / \mathrm{ml}$; Sigma-Aldrich, St. Louis, MO, USA). Ultimately, the accuracy of the cloning and the enzymatic digestion procedures were assessed using PCR and the double-digestion by restriction enzymes NcoI and HindIII (Thermo Fisher Scientific, Boston, MA, USA) at the same time, respectively. Nucleotide sequencing also confirmed this procedure (Bioneer South Korea).

Expression of the rPorA in $E$. coli

To express recombinant proteins, several colonies were randomly inoculated in $50 \mathrm{ml}$ of $\mathrm{LB}$ broth with $50 \mu \mathrm{g} / \mathrm{ml}$ Kanamycin (Merck, Darmstadt, Germany) for the analysis of protein expression until they reached an optical absorption coefficient of 0.5-0.6 at a wavelength of $600 \mathrm{~nm}$. At this point, the cultures were induced with $0.5,1.0,2.0$, and $4.0 \mathrm{mM}$ concentrations of IPTG (Fermentas) and the protein expression was analyzed $4 \mathrm{~h}$ following the induction. Prior to the induction, a sample of the bacterial culture was taken as negative control. Finally, the pellet was resuspended in lysis buffer (urea 8M, NaH2PO4 0.1M, Tris 0.01M pH8.0) and this suspension was sonicated (five cycles, $30 \mathrm{~s}$ each, with intervals of $30 \mathrm{~s}$ on ice) and centrifuged 15 minutes at $6000 \times \mathrm{g}$. The supernatant was added to a chromatography column containing Nickel-nitrilotriacetic acid (Ni-NTA) gel matrix (Qiagen, Hilden,Germany). After $1 \mathrm{~h}$, the proteins bound to agarose were removed by washing and elution buffers and were analyzed by SDS-PAGE.

\section{SDS-PAGE and Western Blotting}

Confirmation of rPorA expression was performed using 15\% SDS-PAGE gel, followed by Western Blotting. The proteins from the gel were transfered to the nitrocellulose membrane and were exposed to HRP-conjugated anti-His Tag monoclonal antibody (Qiagen, Hilden,Germany) at a dilution ratio of 1:6000 for $2 \mathrm{~h}$ at room temperature. The color reaction was ultimately generated in the presence of DAB substrate.

\section{RESULTS}

PCR of porA and cloning of pET28a-porA construct To amplify the porA gene from the genome of $N$. meningitidis serogroup A, PCR was performed under the described conditions. An amplicon with a size of approximately $1200 \mathrm{bp}$, indicating a successful amplification was obtained (Fig .1). The gene construct was digested with $N c o$ I and Hind III and revealed a 1200 bp gene fragment and a 5400bp linear plasmid (Fig. 2).

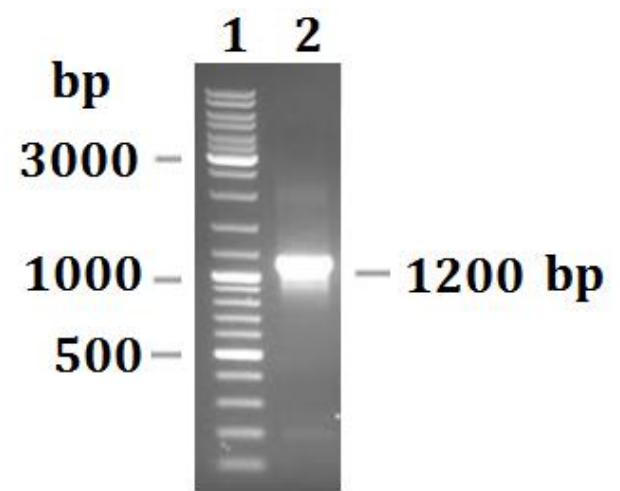

Fig. 1. Amplification of PorA gene by PCR. Lane 1: DNA Ladder Mix Marker (CinnaGen, Iran) . Lane 2: porA gene amplicon (1200 bp).

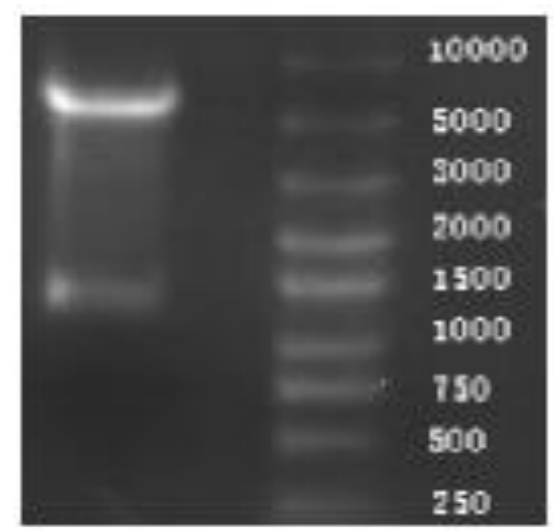

Fig. 2. Double digestion of pET28a- PorA by restriction enzymes NcoI and Hind III.

Verification of rPorA 
SDS-PAGE showed a $45 \mathrm{kDa}$ fragment protein. The protein was best expressed using $2 \mathrm{mM}$ concentration of IPTG after 4 h. Western Blotting confirmed the expression of $45-\mathrm{kDa}$ Histagged rPorA. However, no band was detected in the bacterial cell extract before induction with IPTG and in the bacteria containing the pET-28a vector without the insert (Fig 3 and 4).

\section{1}

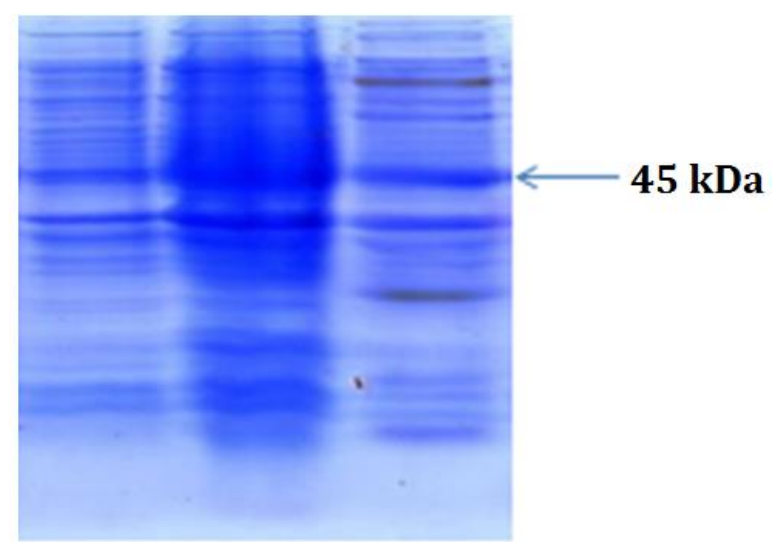

Fig. 3. PorA protein expression induction in the expression system pET28a. Lane 1: Induced clone with $2 \mathrm{mM}$ IPTG (45 kDa). Lane 2: Non-induced clone. Lane 3: Protein Marker.

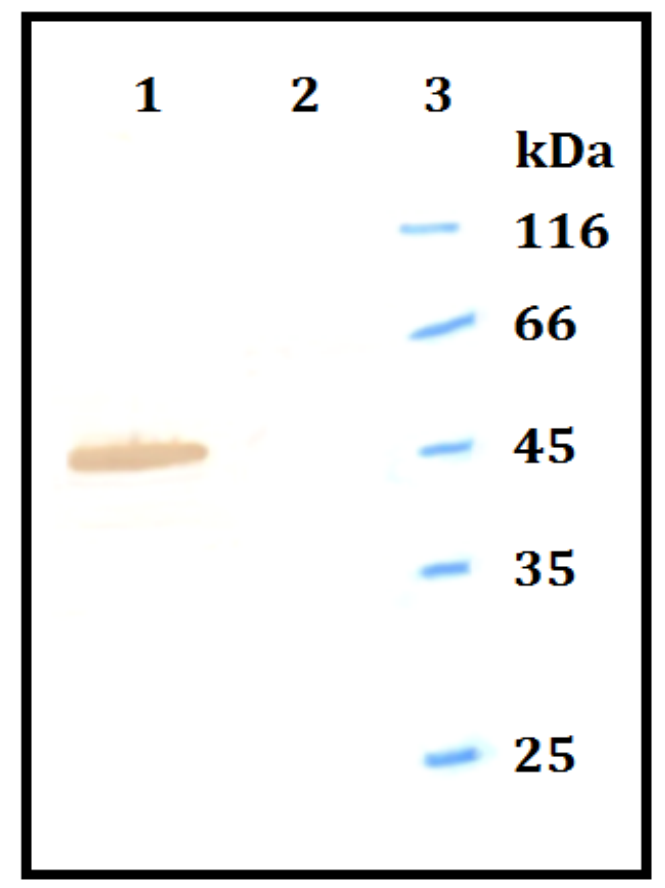

Fig. 4. PorA protein expression by Western blot in the expression system pET28a. Lane3: Protein Marker. Lane 2: No induced clone. Lane1: Induced with $2 \mathrm{mM} / \mathrm{IPTG}$. (45 kDa).

\section{Sequencing and homology}

Nucleotide sequencing and bioinformatics analysis of serogroup A of $N$. meningitidis porA showed $97 \%$ homology compared to the reference gene (NCBI GenBank accession number AL157959.1).

\section{DISCUSSION}

Claassen et al. and Longworth et al. have designed recombinant OMV vaccines with six (Hexamer) or nine (Nanomer) different PorA serosubtype which provides protection against $N$. meningitidis strains. The Hexamer vaccine consists of OMV prepared from two meningococcal strains that express PorA. This vaccine is in phase I of clinical studies and has managed to cover $75 \%$ of the circulating serogroup B strains [14, 15]. Ohallahan et al. have collaborated with the New Zealand Ministry of Health to develop a vaccine based on the strain B:4:P1,7b,4 containing PorB, PorA and Lipo-OligoSaccharides (LOS) proteins. This vaccine was successfully administered to children and newborns in the first and second phases of the project and showed that it can produce bactericidal antibody responses in $75 \%$ of $6-24$ month-old infants and 8-12 year-old children [16]. Moreover, Lusta et al. have shown that PorA can be easily supplied in live bacteria and stimulate bactericidal antibodies [17]. Findlow and colleagues have also investigated the cross-reactivity of antiPorA antibodies following immunization with serogroup B monovalent and hexavalent OMV vaccines through the evaluation of serum bactericidal activity (SBA). They have shown that the hexavalent OMV vaccine can generate crossreactive antibody responses against various strains of the serogroup B [18].

Considering the above and given that PorA is a major protein in $N$. meningitidis OMVs, the present study was conducted to investigate porA cloning and expression in pET28a expression system, as a first step for a PorA vaccine study against $\mathrm{N}$. meningitides strain ATCC CSBPI G243. Our results indicated that pET28a expression system using $2 \mathrm{mM}$ IPTG for $4 \mathrm{~h}$ can be successfully used to produce rPorA. Following optimization of the protein expression conditions, further studies can be conducted to determine the immunogenic potential of this protein in the presence of different adjuvants and the probability of producing immunizing responses. Further studies are necessary for investigating the potential and the efficiency of the protein as a vaccine candidate against infections caused by N. meningitidis.

\section{ACKNOWLEDGEMENT}

This work was supported by a grant (No B9109) from Pasture Institute of Iran (Tehran,Iran).

\section{CONFLICT OF INTEREST}

The authors declare that they have no conflict of interest.

\section{REFERENCES}

1. Tunkel AR, Hartman BJ, Kaplan SL, Kaufman BA, Roos KL, Scheld $\mathrm{WM}$ et al. Practice guidelines for the management of bacterial meningitis. Clinical infectious diseases. 2004;39(9):1267-84.

2. Harrison LH, Trotter CL, Ramsay ME. Global epidemiology of meningococcal disease. Vaccine. 2009;27:B51-B63.

3. Harcourt BH, Anderson RD, Wu HM, Cohn AC, MacNeil JR, Taylor TH et al., editors. Population-based surveillance of Neisseria meningitidis antimicrobial resistance in the United States. Open forum infectious diseases; 2015: Oxford University Press.

4. Adu-bobie J, Pizza M, Norais N, Ferrari G, Grandi G. IMMUNOGENIC BACTERIAL VESICLES WITH OUTER MEMBRANE PROTEINS. US Patent 20,160,108,094; 2016.

5. Adu-Bobie J, Pizza M, Norais N, Ferrari G, Grandi G. Immunogenic bacterial vesicles with outer membrane proteins. Google Patents; 2015. 
6. Delbos V, Lemée L, Bénichou J, Berthelot G, Deghmane A-E, Leroy J-P et al. Impact of MenBvac, an outer membrane vesicle (OMV) vaccine, on the meningococcal carriage. Vaccine. 2013;31(40):4416-20.

7. Hong E, Giuliani MM, Deghmane A-E, Comanducci M, Brunelli B, Dull $\mathrm{P}$ et al. Could the multicomponent meningococcal serogroup $\mathrm{B}$ vaccine (4CMenB) control Neisseria meningitidis capsular group $\mathrm{X}$ outbreaks in Africa? Vaccine. 2013;31(7):1113-6.

8. Peak IR, Jennings CD, Jen FE-C, Jennings MP. Role of Neisseria meningitidis PorA and PorB expression in antimicrobial susceptibility. Antimicrobial agents and chemotherapy. 2014;58(1):614-6.

9. Peak IR, Chen A, Jen FE, Jennings C, Schulz BL, Saunders NJ et al. Neisseria meningitidis lacking the major porins PorA and PorB are viable and modulate apoptosis and the oxidative burst of neutrophils. Journal of proteome research. 2015.

10. Kaaijk P, van Straaten I, van de Waterbeemd B, Boot EP, Levels LM, van Dijken $\mathrm{HH}$ et al. Preclinical safety and immunogenicity evaluation of a nonavalent PorA native outer membrane vesicle vaccine against serogroup B meningococcal disease. Vaccine. 2013;31(7):1065-71.

11. Nagaputra JC, Rollier CS, Sadarangani M, Hoe JC, Mehta OH, Norheim $\mathrm{G}$ et al. Neisseria meningitidis native outer membrane vesicles containing different lipopolysaccharide glycoforms as adjuvants for meningococcal and nonmeningococcal antigens. Clinical and Vaccine Immunology. 2014;21(2):234-42.

12. Veyrier FJ, Williams AH, Mesnage S, Schmitt C, Taha MK, Boneca IG. De-O-acetylation of peptidoglycan regulates glycan chain extension and affects in vivo survival of Neisseria meningitidis. Molecular microbiology. 2013;87(5):1100-12
13. Takao K, Miyakawa T. Genomic responses in mouse models greatly mimic human inflammatory diseases. Proceedings of the National Academy of Sciences. 2015;112(4):1167-72.

14. Longworth L, Brazier J, Yang Y. EQ-5D in skin conditions: an assessment of validity and responsiveness. 2014.

15. Claassen I, Meylis J, van der Ley P, Peeters C, Brons H, Robert J et al. Production, characterization and control of a Neisseria meningitidis hexavalent class 1 outer membrane protein containing vesicle vaccine. Vaccine. 1996;14(10):1001-8.

16. Sexton K, Lennon D, Oster P, Crengle S, Martin D, Mulholland K et al The New Zealand Meningococcal Vaccine Strategy: a tailor-made vaccine to combat a devastating epidemic. The New Zealand Medical Journal (Online). 2004;117(1200).

17. Lusta K. Bacterial outer membrane nanovesicles: Structure, biogenesis, functions, and application in biotechnology and medicine (Review) Applied Biochemistry and Microbiology. 2015;51(5):485-93.

18. Findlow J, Borrow R, Snape MD, Dawson T, Holland A, John TM et al. Multicenter, open-label, randomized phase II controlled trial of an investigational recombinant meningococcal serogroup $B$ vaccine with and without outer membrane vesicles, administered in infancy. Clinical Infectious Diseases. 2010;51(10):1127-37. 